\title{
OPTICAL PROPERTIES OF PARTICLES
}

\section{IN PLANETARY ATMOSPHERES: LABORATORY STUDIES}

\author{
DUDLEY WILLIAMS \\ Kansas State University, Manhattan, Kan., U.S.A.
}

\begin{abstract}
The laboratory techniques employed for the determination of optical constants are discussed briefly. The values obtained for the real $n$ and imaginary $k$ (or $n_{i}$ ) parts of the refractive indices of water, ice, and liquid ammonia are presented graphically. Work currently in progress on solid ammonia and on sulphuric acid is discussed. Other approaches to studies of the properties of aerosols in planetary atmospheres are presented briefly.
\end{abstract}

\section{Introduction}

Most of our information concerning the nature of planetary atmospheres and planetary surfaces has been derived from studies of the reflection and scattering of incident solar radiation by the planets. With the development of infrared methods, the thermal emission spectra of planets have provided additional information. Any quantitative interpretation of the spectral reflection or spectral emission by the planets depends upon not only a detailed knowledge of the spectral characteristics of their atmospheric gases but also a knowledge of the real $n(v)$ and imaginary $k(v)$ or $n_{i}(v)$ parts of the refractive indices of the particles of cloud or dust in their atmospheres. If these latter optical properties are known, this theory can be applied to observed spectra to give estimates of the number density, the range of sizes, and the spatial distribution of the particles in planetary atmospheres; in cases of dense cloud cover, this knowledge can, in turn, provide important information regarding planetary heat balances.

Although much spectroscopic work has been done on many of the substances that may be present in planetary aerosols, a survey by Irvine and Pollack (1968) revealed that even in the case of the most abundant telluric aerosols, water and ice, published spectra did not form the basis for determining accurate values of $n(v)$ and $k(v)$ in much of the infrared. Since the time of the Irvine-Pollack survey, extensive quantitative infrared work has been done on water, ice, the condensed phases of ammonia, and on sulphuric acid, all of which exist as planetary aerosols.

\section{Methods of Determining Optical Constants}

The general methods of determining optical constants $n(v)$ and $k(v)$ have been treated definitively by Humphrey-Owen (1961). They may be determined from measurements of the reflectance of polarized radiation at two angles of incidence (Querry et al., 1969) and subsequent solution of the generalized Fresnel equations (Querry, 1969). They can also be determined from measurements of fractional spectral transmittance $T(v)$ of a layer of thickness $x$ which give the Lambert absorption coefficient $\alpha(v)$ from 
the relation

$$
T(v)=\exp [-\alpha(v) x]
$$

together with measurements of the reflectance of polarized radiation at a single angle of incidence or with measurements of the reflectance of unpolarized radiation at nearnormal incidence. In the latter case $k(v)=\alpha(v) / 4 \pi v$, where $v$ is expressed in $\mathrm{cm}^{-1}$, and $n(v)$ can be subsequently determined from the simple relation

$$
R(v)=\frac{[n(v)-1]^{2}+k(v)^{2}}{[n(v)+1]^{2}+k(v)^{2}}
$$

where $R(v)$ is the fractional reflectance at near-normal incidence.

Alternatively, Kramers-Kronig theorems can be applied in cases where $\alpha(v)$ has been measured over a large spectral interval from the relation

$$
n(v)=1+\left(1 / 2 \pi^{2}\right) P \int_{0}^{\infty} \frac{\alpha\left(v^{\prime}\right)-\alpha(v)}{v^{\prime 2}-v^{2}} \mathrm{~d} v^{\prime} .
$$

In using Equation (3) the values of $n(v)$ can be determined in excellent approximation inside the spectral range of measurement provided one makes reasonable assumptions regarding $\alpha(v)$ outside the range of measurement (Robertson et al., 1973); Equation (3) provides exact values of $n(v)$ only if $\alpha(v)$ is known for all frequencies. In other cases where $R(v)$ has been measured over a large spectral interval, both $n(v)$ and $k(v)$ can be obtained within the spectral range of measurement by use of Kramers-Kronig phase-shift analysis (Robertson et al., 1973). According to this Kramers-Kronig theorem, if the modulus $[R(v)]^{1 / 2}$ of the complex reflectivity $[R(v)]^{1 / 2} e^{i \phi(v)}$ is known for all frequencies, the phase shift $\phi(v)$ at frequency $v$ is given by

$$
\phi(v)=\frac{2 v_{0}}{\pi} P \int_{0}^{\infty} \frac{\ln [\mathrm{R}(v)]^{1 / 2}}{v^{2}-v^{\prime 2}} \mathrm{~d} v^{\prime} ;
$$

$n(v)$ and $k(v)$ can then be determined from $\phi(v)$. Even though $R(v)$ is not known for all frequencies, Equation (4) gives values of $\phi(v)$ within the range of measurement (Hale et al., 1972) that are not strongly influenced by assumed values of $R(v)$ outside the range of measurement. In the case of water, there is excellent agreement between values of optical constants determined by the methods just described (Querry, 1969; Robertson et al., 1973; Hale et al., 1972; Rusk et al., 1971; Pontier and Dechambenoy, 1965-1966; Zolatarev et al., 1969).

In using any of the computation methods just described, it is necessary that extreme care be taken in the experimental determination of $\alpha(v)$ and $R(v)$. In determining $\alpha(v)$ for a liquid in the infrared, measurements of the intensity $I_{0}(v)$ of the incident radiation and the intensity $I(v)$ of the radiation transmitted through an absorption 
cell containing a liquid layer of thickness $x$ lead to the relation

$$
T(v)=[1-\mathfrak{R}(v)][1-\mathfrak{A}(v)] \exp [-\alpha(v) x],
$$

where $\Re(v)$ is the fraction of the incident radiation reflected at the inner and outer surfaces of the cell windows and $\mathfrak{A}(v)$ is the fraction of the incident radiation absorbed by the cell windows; $\mathfrak{R}(v)$ and $\mathfrak{A}(v)$ cannot be easily measured. Further difficulties are introduced by the required values of $x$ as illustrated for the case of water by the curves in Figure 1, in which the main curve represents the fractional spectral transmittance $T(v)$ for a layer of liquid only $10 \mu \mathrm{m}$ thick; it will be noted that a water layer of this thickness is essentially opaque in the $3400 \mathrm{~cm}^{-1}$ region. In order to deter-

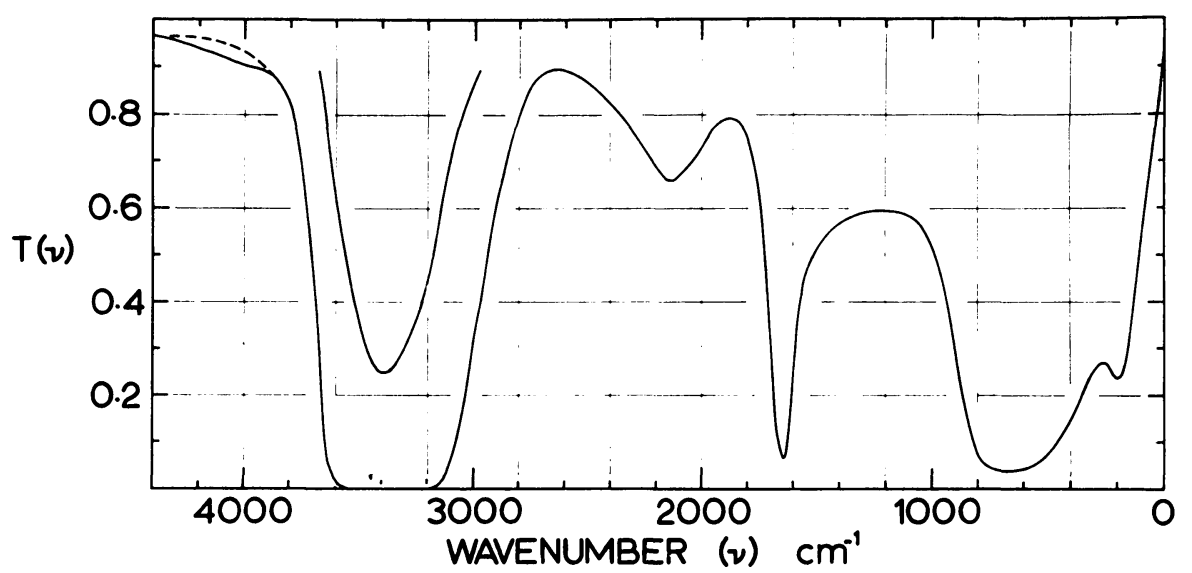

Fig. 1. The spectral transmittance $T(v)$ of a $10 \mu \mathrm{m}$ layer of water in the infrared; the upper curve gives $T(v)$ for a $1.125 \mu \mathrm{m}$ layer in the vicinity of $3400 \mathrm{~cm}^{-1}$.

mine $\alpha(v)$ in the $3400 \mathrm{~cm}^{-1}$ region, it would be necessary to prepare a uniform layer of thickness $1.125 \mu \mathrm{m}$, which would give the spectral transmittance given by the upper curve in the $3400 \mathrm{~cm}^{-1}$ region. Fortunately, Robertson and Williams (1971) have developed a technique involving a wedge-cell of thickness ranging from optical contact at one edge to $20 \mu \mathrm{m}$ at the other edge; by passing such a cell laterally through the beam of radiation and measuring values of $T(v)$ at various positions corresponding to various thicknesses of the absorbing layer, one can eliminate the effects of $\mathfrak{R}(v)$ and $\mathfrak{A}(v)$ by taking ratios of $T(v)$ for different thicknesses; the thicknesses of the wedge-shaped layer can be measured and monitored by interferometric techniques.

In the determination of absolute values of $R(v)$ we have adopted a technique of first measuring the nominal reflectance of a surface relative to a front surface reference mirror. The absolute spectral reflectance of the reference mirror is carefully determined by an independent measurement involving a reflectometer of a type developed by Strong (1938). From the two sets of experimental measurements, absolute values of $R(v)$ can be obtained.

Since the values of $R(v)$ in most spectral regions are small, care must be taken to 
see that detector-amplifier-recorder systems are strictly linear in giving chart deflections that are strictly proportional to the radiant flux reaching the detector. Rather than relying on changes in amplifier gain settings, we have made use of optical attenuators consisting of rapidly rotating calibrated sector wheels inserted in the beam when the reference mirror is being used. Under these conditions we have found that thermocouples used with conventional amplifiers with fixed gain controls give satisfactorily linear response.

\section{Optical Constants}

The most abundant particulate constituent of the Earth's atmosphere is liquid water in the form of cloud droplets. The optical constants of water at $25^{\circ} \mathrm{C}$ are shown in Figures 2 and 3 ; the values of these constants have been tabulated in earlier published work (Robertson et al., 1973; Hale et al., 1972; Rusk et al., 1971; Pontier and

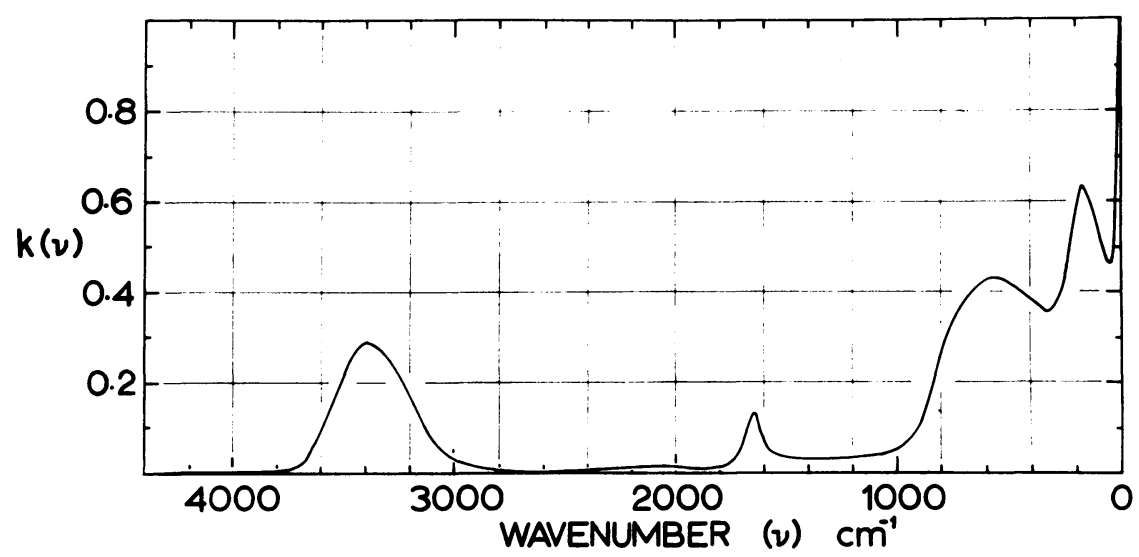

Fig. 2. The imaginary part $k(v)$ of the complex index of refraction $N(v)=n(v)+i k(v)$ of water at $25^{\circ} \mathrm{C}$.

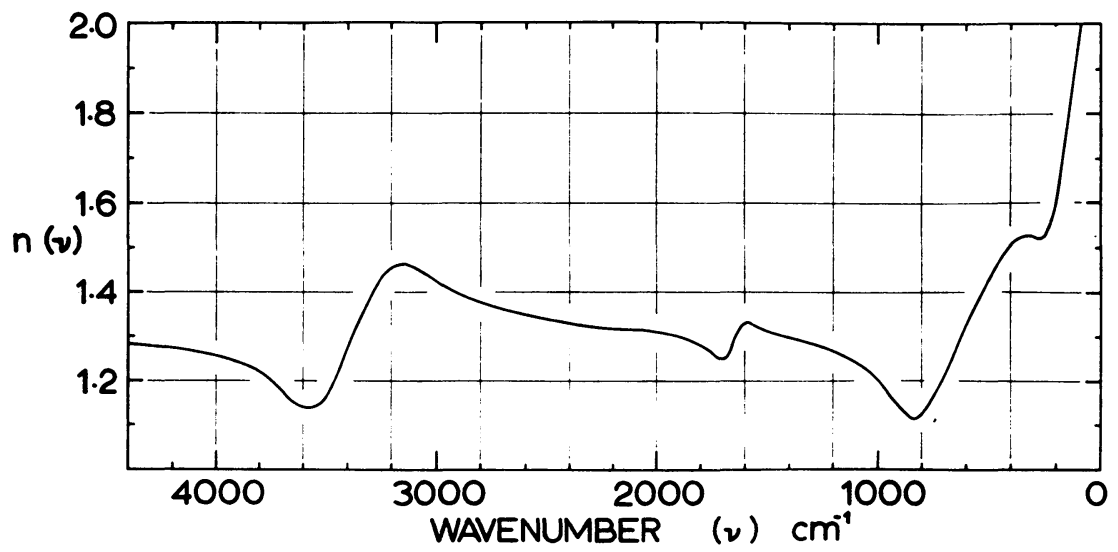

Fig. 3. The real part $n(r)$ of the complex index of refraction $N(v)=n(r)+i k(v)$ of water at $25^{\circ} \mathrm{C}$. 
Dechambenoy, 1965-1966; Zolatarev et al., 1969). The influence of temperature on the optical constants of water has been investigated by Hale et al. (1972). Optical constants in the submillimeter region have been determined by Davis et al. (1970) and by Zafar et al. (1973). Also present as an abundant aerosol in the Earth's atmosphere are ice crystals; the reflection spectrum of ice has recently been investigated by Schaaf and Williams (1973), whose values of the optical constants based on Kramers-Kronig phaseshift analysis are plotted in Figures 4 and 5. Ice particles have recently been detected in the Martian spectrum in the course of the recent Mariner flight.

Ammonia in condensed states is probably an important particulate constituent of the cloud cover of the major planets. The reflection spectrum of liquid ammonia has been measured in the visible and in the infrared by Robertson and Williams (1973a, b), who have also measured the transmission spectrum in the near infrared; because of the lack of suitable cell-window materials, these workers were unable to obtain transmission data in the far-infrared. Optical constants based on these studies of liquid ammonia are plotted in Figures 6 and 7. Robertson and Williams (1973) have also attempted to

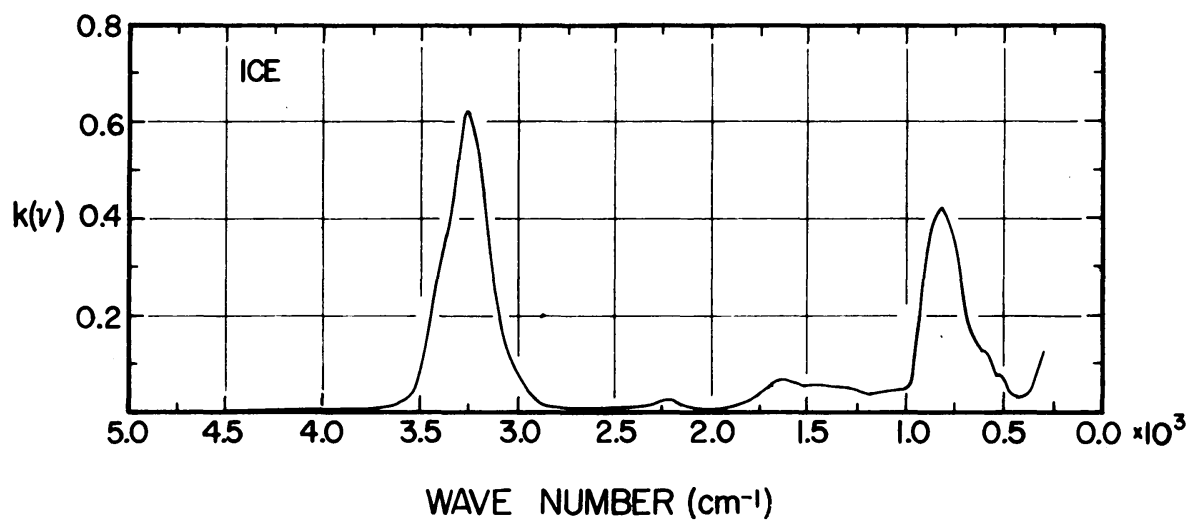

Fig. 4. The imaginary part $k(v)$ of the complex refractive index of ice at $-7^{\circ} \mathrm{C}$.

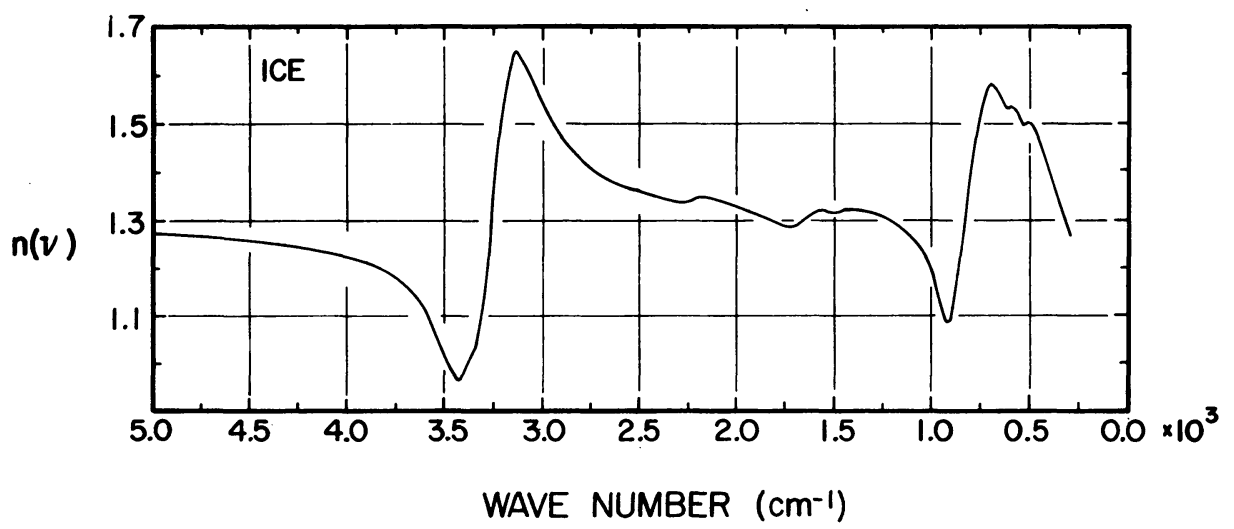

Fig. 5. The real part $n(v)$ of the complex refractive index of ice at $-7^{\circ} \mathrm{C}$. 
determine the optical constants of solid ammonia in the infrared at a temperature slightly below the melting point; they have succeeded in obtaining satisfactory transmission spectra in the $5000-900 \mathrm{~cm}^{-1}$ region in the near infrared. However, because of the high vapor pressure of solid ammonia, they have thus far been unsuccessful in obtaining acceptable reflection spectra; a good reflecting surface can be maintained for only brief periods. Other techniques for studying solid ammonia are being investigated.

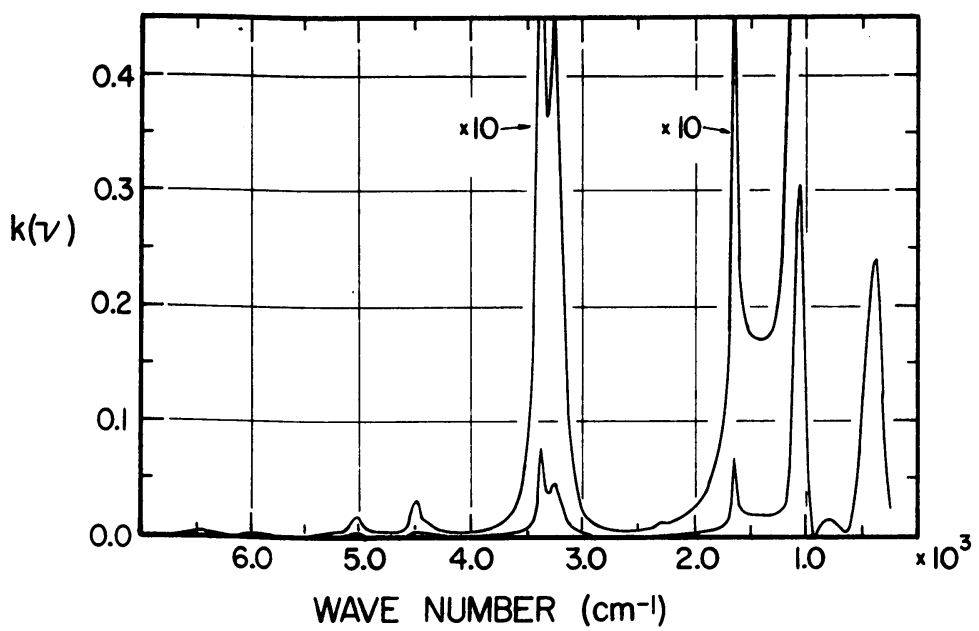

Fig. 6. The imaginary part $k(v)$ of the complex refractive index of liquid ammonia at $-45^{\circ} \mathrm{C}$.

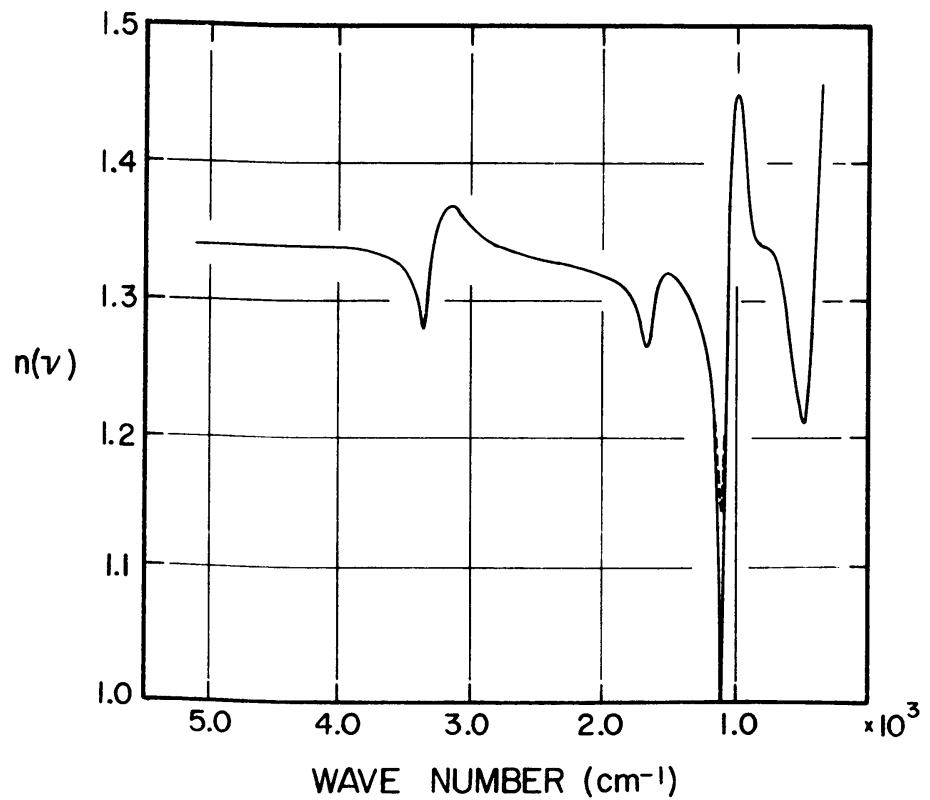

Fig. 7. The real part $n(v)$ of the complex refractive index of liquid ammonia at $-45^{\circ} \mathrm{C}$. 
The most abundant aerosol in the Earth's stratosphere consists of sulphuric acid droplets; it has also been suggested that sulphuric acid in liquid form is the dominant constituent of the clouds of Venus (Young, 1973). Palmer (1973) and Williams have measured the spectral reflectance of sulphuric acid in aqueous solution at a number of concentrations in the visible and intermediate infrared; transmission measurements are in progress in the near-ultraviolet-visible-near-infrared-region. The results of this study should provide values that will be useful in detailed studies of the clouds of Venus. Preliminary values of the optical constants for sulphuric acid solutions in the infrared, based on Kramers-Kronig phase-shift analysis of reflectance measurements, have been obtained; improved values based on our study will soon be available. Other reflection data providing values of $n(v)$ and $k(v)$ have been obtained by Querry and his colleagues (1974) for a 3-M solution of sulphuric acid. Remsberg (1973) has used attenuated-total-reflection (ATR) techniques to obtain the optical constants for 95 and $75 \%$ solutions of sulphuric acid in the $770-1250 \mathrm{~cm}^{-1}$ region, which covers an important window in the Earth's atmosphere. On the basis of a preliminary analysis of spectra taken from high-altitude aircraft, Pollack (1973) indicates that the spectrum in the $2-5 \mu \mathrm{m}$ region of the Venus clouds resemble spectra reported for $75 \%$ sulphuric acid.

\section{Actual Particles in Planetary Atmospheres}

We recognize that planetary cloud covers contain numerous types of particles that are not composed of pure chemical substances of the type we have investigated; for example, in the case of the Earth's atmosphere, many of the water droplets over the sea are formed on salt nuclei, and many other types of aerosols are known to be present. In the case of the Earth and even more so in the case of Mars, dust storms can occasionally contribute to the particulate content of the atmosphere. In the case of the major planets, compounds other than pure ammonia are believed to contribute to the cloud cover. Thus, optical studies of pure substances can at best contribute only rough guides to an interpretation of astronomical observations.

Since the number of possible mixtures of materials in planetary cloud covers is infinite, it would appear pointless to attempt laboratory measurements of the 'effective optical constants' of various mixtures that have been suggested for the aerosol content of the atmospheres of various planets. Two courses appear possible; the first of these would involve mathematical combinations of the optical constants of pure substances to obtain values for 'effective optical constants' for various mixtures; the second course would be the careful selection of a limited set of materials believed to be present and to make careful laboratory studies of these selected materials.

An important example of this second course is the study (Pollack et al., 1973) of a set of carefully selected terrestrial rocks that might be present in abundance in planetary surfaces and therefore in the atmospheric dust of planets. Rocks and most minerals in the Earth's crust vary in composition with locality; thus careful selection of specimens is also of great importance. Another example of the second course is the work of Volz $(1972,1973)$ who has obtained optical constants for aerosols of types actually 
collected in the Earth's atmosphere; this method will become possible for other planets only after particles have been collected and brought back to Earth. However, it may ultimately become of great importance in detailed studies of planetary heat balances. Meanwhile, we hope that our laboratory studies of pure materials will be of some use in the interpretation of observations.

\section{Acknowledgements}

We should like to express our appreciation to the U.S. National Aeronautics and Space Administration and to the U.S. Office of Naval Research, which have supported our work. Our thanks also go to Dr J. B. Pollack of the NASA Ames Research Center for assistance in our work on sulphuric acid and to Dr Andrew T. Young of the Jet Propulsion Laboratory for helpful discussions.

\section{References}

Davies, M., Pardoe, G. W. F., Chamberlain, J., and Gebbie, H. A.: 1970, Trans. Faraday Soc. 66, 273.

Hale, G. M., Querry, M. R., Rusk, A. N., and Williams, D.: 1972, J. Opt. Soc. Am. 62, 1103.

Humphrey-Owens, S. P. F.: 1961, Proc. Roy. Soc. London 77, 949.

Irvine, W. H. and Pollack, J. B.: 1968, Icarus 8, 324.

Palmer, K.: 1973, Proc. Symposium on Molecular Spectra, Ohio State Univ., p. 168.

Pollack, J. B.: 1973, private communication.

Pollack, J. B., Toon, O. B., and Khare, B. N.: 1973, Icarus 19, 373.

Pontier, L. and Dechambenoy, C.: 1965, Ann. Geophys. 21, 462.

Pontier, L. and Dechambenoy, C.: 1966, Ann. Geophys. 22, 633.

Querry, M. R.: 1969, J. Opt. Soc. Am. 59, 876.

Querry, M. R., Curnutte, B., and Williams, D.: 1969, J. Opt. Soc. Am. 59, 1299.

Querry, M. R., Waring, R. C., Holland, W. E., Earls, L. M., Herman, M. D., Nijm, W. P., and Hale, G. M.: 1974, J. Opt. Soc. Am. 44, 39.

Remsberg, E. E.: 1973, J. Geophys. Res. 78, 1401.

Robertson, C. W.: 1973, Proc. Symposium on Molecular Spectra, Ohio State Univ., p. 167.

Robertson, C. W., Curnutte, B., and Williams, D.: 1973, Mol. Phys. 66, 183.

Robertson, C. W. and Williams, D.: 1971, J. Opt. Soc. Am. 61, 1316.

Robertson, C. W. and Williams, D.: 1973a, J. Opt. Soc. Am. 63, 763.

Robertson, C. W. and Williams, D.: 1973b, J. Opt. Soc. Am. 63, 188.

Rusk, A. N., Querry, M. R., and Williams, D.: 1971, J. Opt. Soc. Am. 61, 895.

Schaaf, J. W. and Williams, D.: 1973, J. Opt. Soc. Am. 63, 726.

Strong, J.: 1938, Procedures in Experimental Physics, Prentice-Hall, New York, p. 376.

Volz, F. E.: 1972, Appl. Opt.11, 755.

Volz, F. E.: 1973, Appl.Opt. 12, 564.

Young, A. T.: 1973, Icarus 18, 564.

Zafar, M. S., Hasted, J. B., and Chamberlain, J.: 1973, Nature Phys. Sci. 243, 106.

Zolatarev, V. M., Mikhailov, B. A., Aperovich, L. I., and Popov, S. I.: 1969, Opt. Spectrosk. $27,790$.

\section{DISCUSSION}

Irvine: How temperature dependent are the optical constants of $\mathrm{NH}_{3}$ ? Would your results be applicable to Jovian conditions?

Williams: The present data could probably be extrapolated to Jovian conditions.

Owen: The upper clouds on Jupiter are most likely solid ammonia: but to study liquid droplets, it 
would be more appropriate to study $\mathrm{NH}_{4} \mathrm{OH}, \mathrm{NH}_{4} \mathrm{SH}$ or $\left(\mathrm{NH}_{4}\right)_{2} \mathrm{~S}$, since the lower, liquid droplet clouds at $T=225 \mathrm{~K}$ will not be pure $\mathrm{NH}_{3}$.

Williams: The optical qualities of $\mathrm{NH}_{4} \mathrm{OH}$ are at present under study in our laboratory.

Huebner: Do you have, or plan to obtain, frequency-dependent data of the complex refractive index for the refractory compounds like enstatite? These are important for interplanetary dust and meteoric matter.

Gulkis: A previous comment from the floor stated that the reflecting cloud temperature is about $225 \mathrm{~K}$. The ammonia cloud and haze regions, however, form at $150 \mathrm{~K}$. At higher temperatures one expects to find a solution or droplets of water and ammonia rather than ammonia ice. 